\section{Less shocking antitumor agents?}

Salmonella possesses intriguing tumor-homing properties, but the toxic inflammatory responses induced by its lipopolysaccharides (most notably lipid A) have made it less attractive as an anticancer agent. Using attenuated strains of Salmonella previously shown to target and invade tumors, Low et al. have now engineered strains with a disrupted $m s b B$ gene that induce markedly reduced levels of tumor necrosis factor (TNF) $-\alpha$ and are 10,000-fold less toxic to mice (see pp. 19 and 37. This suggests that tumor-targeting strains of Salmonella can be created that retain tumor-retarding properties, but exhibit reduced side effects such as septic shock.

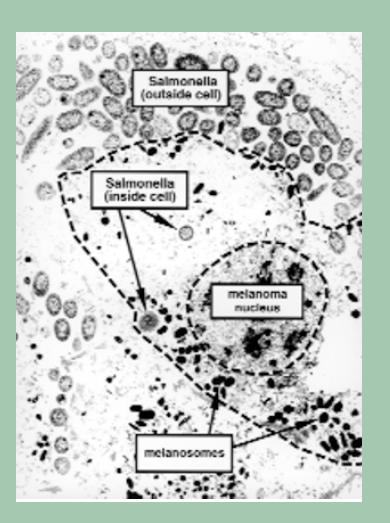

\section{Ribozymes as diagnostics}

Catalytic RNAs, or ribozymes, have received significant interest as potential therapeutic agents; however, their use as diagnostics has remained largely unexplored. In this issue, novel ligase ribozymes are selected that are 10,000-fold more active in the presence of a cDNA primer or "effector." After optimization by mutagenesis, these ribozymes were engineered with an RNA ATP-binding sequence that allowed activity to be influenced by ATP. When linked with reverse transcriptase-PCR, the authors show that the ribozymes can be used to quanitify both oligonucleotides or small-molecule analytes (seep. 62 .

\section{Rapid histological analysis}

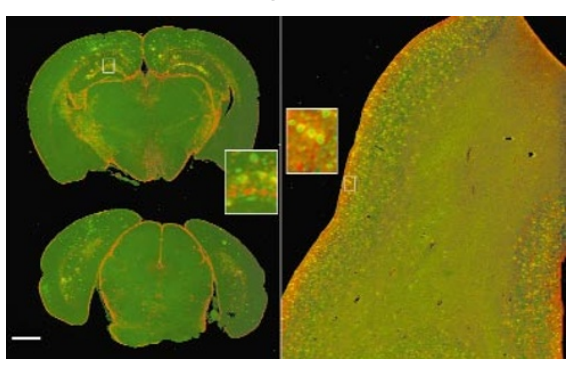

The microscope has played a central role in histological analysis. Now, an alternative technique, originally designed for DNA microarray analysis, has been adapted for use in the rapid and quantitative analysis of histological specimens. Hanzel et al. compare the performance of a confocal laser fluorescence microscanner with that of a conventional microscope, studying immunostained amyloid plaques from Alzheimer's patients and brain sections from a transgenic model of Alzheimer's. They found that the scanner has a larger field of view, more uniform response, superior light collection, and larger dynamic range than traditional microscopic analysis (see p. 53.

Research Briefs written by Andrew Marshall.

\section{Photoreversible enzyme solubility}

The ability to control enzyme solubility is important for optimizing catalytic efficiency in organic solvents and to recover enzymes from solution at the end of a chemical reaction. Ito et al. shed some light on the problem by coupling to subtilisin a photoresponsive copolymer that allows solubility to be controlled by photoirradiation. This hybrid subtilisin could be recovered from solution after ultraviolet irradiation and resolubilized by visible light, even after several cycles of precipitation and solubilization (see p. 73).

\section{Antisense that delivers}

A longstanding problem in antisense research is getting the oligonucleotide to the target of interest within the nucleus. A novel phenoxazine-substituted 7-mer oligonucleotide is described in this issue that exhibits an enhanced ability to permeate cells in culture and migrates to the nucleus without the assistance of delivery agents. This modified oligonucleotide has a higher binding affinity to a complementary RNA target than its parent molecule, showed similar antisense potency, and is taken up by the nucleus in a variety of cell types (seep. 48 .

\section{A trick in the tail}

A paper on p. 58 stretches the possibilities for molecular evolution. Urabe and colleagues provide the first description of an approachtermed random elongation mutagenesis-in which peptide tails containing randomized sequences are appended to the $\mathrm{C}$ terminus of enzymes. Using catalase I enzyme from Bacillus stearothermophilus as a test system, they isolated 15 Escherichia coli clones from 58 clones that had higher thermostability than the wild type, a significant achievement given that catalase was considered to be "optimized" (see also p. 21).

\section{Four goes into three}

When it comes to fixing carbon, crop plants such as rice are at a distinct disadvantage. They use the $\mathrm{C}_{3}$ photosynthetic pathway, which is much more sensitive to $\mathrm{O}_{2}$ inhibition than plants that directly produce $\mathrm{C}_{4}$ dicarboxylic acids from $\mathrm{CO}_{2}$ via an enzyme called phophoenolpyruvate carboxylase (PEPC). In this issue, researchers have successfully transformed rice with the PEPC gene from the $\mathrm{C}_{4}$ plant maize. While this represents a preliminary step in engineering a $\mathrm{C}_{4}$ pathway, introduction of the PEPC transgene into rice was sufficient to reduce $\mathrm{O}_{2}$ inhibition of photosynthesis compared with wildtype plants (see pp. 22 and 76 ).

Phage display has been widely applied to express diverse libraries of proteins or peptides with desirable properties. In this issue, Legendre et al. add a new application for phage display-the design of molecular sensors based on the modulation of

enzyme activity by

monoclonal antibodies

(Mabs). They intro-

duced random pep-

tide libraries into

three loops close

to the active

site of $\beta$-lact-

amase and selected vari-

ants that

showed al-

tered activity

in the presence

of three

different antiprostate specific

antigen (PSA) monoclonal antibodies (Mabs). In a competitive assay, the altered enzyme was capable of detecting PSA at a minimal concentration of $10^{-9} \mathrm{M}$. Such Mab-regulated enzymes may be useful in the design of diagnostics for many different analytes, particularly as the approach has no requirement for knowledge of the natural epitope (see p. 67).

One way of combating pathogens is to prevent them from gaining a foothold at the site of infect-

ion. With this in mind, Kelly et al. have designed an antimicrobial peptide to mimic the adhesin of the pathogen Streptococcus mutans-one of the major causes of dental caries. This peptide suppresses adin vitro and patien were protected from streptococcal recolonization (see pp. 20 and 42). 